ORIGINAL

\title{
Composición bromatológica de la carne de conejos suplementados con mataratón y cachaza de palma aceitera
}

\section{Bromatological composition of rabbit meat supplemented with mataraton and palm-press fiber}

\author{
Auristela Malavé A, ${ }^{1 *}$ Ph.D, Luis Córdova $\mathrm{R}^{2}$ Lic, Arlene García $\mathrm{R},{ }^{1} \mathrm{TSU}$, \\ Jesús Méndez $\mathrm{N},{ }^{3}$ M.Sc.
}

\begin{abstract}
${ }^{1}$ Universidad de Oriente, Laboratorios de Investigación "Campus Juanico", Maturín. 2Universidad de Oriente, Programa de Tecnología de Alimentos, Escuela de Zootecnia, "Campus Los Guaritos". 3Universidad de Oriente, Departamento de Agronomía, Escuela de Ingeniería Agronómica, "Campus Los Guaritos", Maturín, Venezuela. *Correspondencia: aumalave@udo.edu.ve.
\end{abstract}

Recibido: Mayo de 2012; Aceptado: Diciembre de 2012.

\section{RESUMEN}

Objetivo. Evaluar comparativamente el efecto de la suplementación del alimento balanceado comercial (ABC) con follaje de mataratón (Gliricidia sepium) y cachaza de palma aceitera (Elaeis guineensis) en la composición bromatológica de la carne de conejo. Materiales y métodos. Las muestras de carne estudiadas en el presente trabajo, provienen de una investigación previa con un diseño experimental de bloques al azar con tres tratamientos (dietas) y tres repeticiones (bloques) con muestreo de tres réplicas por repetición, donde se utilizaron 27 conejos machos mestizos durante el período postdestete divididos en tres tratamientos: uno control $\left(T_{0}\right)$, alimentados sólo con $A B C$, y dos suplementados con mataratón y cachaza de palma aceitera en proporciones de 30 y $10 \%\left(T_{1}\right)$, y 10 y $30 \%\left(T_{2}\right)$, respectivamente, a manera de comparar el efecto de las dietas en el valor nutricional. Resultados. Las muestras de carne provenientes de los diferentes animales en tratamiento, se evaluaron encontrando que la suplementación no afectó significativamente la composición bromatológica $(p>0.05)$ para el contenido de humedad (70.77 a $72.42 \%$ ), proteínas $(19.08$ a $20.34 \%)$, cenizas $(1.53$ a $1.68 \%)$ y lípidos (6.48 a $7.23 \%$ ); indicando que indistintamente de la dieta empleada, suplementando el $A B C$ con mataratón/fibra de palma o no, las carnes de conejo obtenidas son nutricionalmente idénticas como alimento. Conclusiones. Con base en los resultados, se sugiere que el follaje de mataratón y la fibra de palma aceitera podrían constituir una alternativa como recursos agronómicos tropicales en la producción de carne de conejo para el consumo humano.

Palabras clave: Alimentación suplementaria, análisis de alimentos, carne, conejos (Fuente:CAB). 


\begin{abstract}
Objective. Comparatively evaluate the effect of supplementing commercial balanced feed (BCF) with mataratón (Gliricidia sepium) foliage and palm-press fiber (Elaeis guineensis) on the bromatologic composition of rabbit meat. Materials and methods. The meat samples studied came from a previous research which used a randomized block design with three treatments (diets) and three replications (blocks) with three replays by replication sampling where 27 hybrid male rabbits during their post weaning growth period, were used in three treatments: control $\left(\mathrm{T}_{0}\right)$, animals supplied only with $\mathrm{BCF}$, and two supplemented treatments with mataratón foliage and palm-press fiber at 30 and $10 \%\left(T_{1}\right)$, and 10 and $30 \%\left(T_{2}\right)$ proportions, respectively, in order to compare the nutritional value of the different diets. Results. The evaluation of meat samples from different animals in the treatments showed that the bromatological composition of the meat was not significantly affected by the supplementation ( $p>0.05)$ for humidity $(70.77$ to $72.42 \%)$, protein $(19.08$ to $20.34 \%)$, ash (1.53 to $1.68 \%$ ), and lipid (6.48 to $7.23 \%$ ) contents; indicating that, regardless of the diet used, supplementing CBF with mataraton/palm-press fiber or not, the rabbit meats obtained are nutritionally identical. Conclusions. Based on the results, it is suggested that the foliage of mataraton and palm-press fiber may be an alternative, as a tropical agronomic resource, in the rabbit meat production for human consumption.
\end{abstract}

Key words: Food analysis, meat, rabbits, supplementary feeding (Source:CAB).

\section{INTRODUCCIÓN}

Hoy en día, está bien establecido que una alimentación adecuada desempeña un importante papel tanto en el mantenimiento de la salud como en la prevención de las enfermedades, lográndose progresos en la implementación de óptimas estrategias dietéticas (1-3). En este sentido, es necesaria no sólo la búsqueda de nuevas fuentes de alimentos para el consumo humano; sino además, es indispensable conocer la calidad nutricional de las mismas para su posible producción a gran escala.

A lo largo de los años, la carne ha constituido uno de los principales alimentos proveedores de nutrientes tales como proteínas, lípidos, vitaminas, entre otros. Sin embargo, hoy en día existen controversias con respecto a su rol nutricional, debido a que los consumidores consideran que su ingesta en cantidades elevadas está correlacionada con problemas de salud, como obesidad y enfermedades cardiovasculares, por lo que han reducido su consumo (4). Por tanto, muchas personas tienden a modificar su estilo de vida en función de explorar nuevos hábitos dietéticos saludables donde las carnes blancas constituyen una elección favorable, dentro de las cuales la carne de conejo (Oryctolagus cuniculus) se destaca como una valiosa alternativa dietética a nivel nutricional y saludable (5-7).

Durante la última década, las características nutricionales de la carne de conejo han sido evaluadas por diferentes autores a fin de resaltar sus grandes bondades como componente de la dieta (8-10). Con respecto a las carnes rojas y blancas, la carne de conejo es una carne blanca de buen sabor, fácil digestión, con niveles elevados en proteínas y bajos en colesterol, sodio y lípidos con mayor proporción de ácidos grasos insaturados (11), con un valor energético similar al de raciones de las diferentes carnes rojas consumidas comúnmente (12). Adicionalmente, existen estudios relacionados con el alto valor de sus proteínas, como fuente de aminoácidos esenciales $(6,7)$, y con sus propiedades sensoriales que incluyen sabor, textura y color $(10,12)$. Otra de sus características ofrecida a los consumidores, es que no contiene ácido úrico siendo una carne baja en purinas (13). No obstante, a pesar de todas estas cualidades beneficiosas de la carne de conejo, hasta ahora en muchos países sigue siendo deficiente la información que la catalogan como uno de los alimentos de gran interés tanto en la nutrición como en la salud humana $(5,6,14)$.

A lo largo de los últimos años, la incorporación de nuevas alternativas agronómicas por cultivos mejor adaptados al medio, que no son requeridos para la alimentación humana, ha ganado gran atención como recursos utilizables en la alimentación animal y adecuadas a condiciones locales en muchos países del mundo con el fin de abaratar costos, diversificar las opciones alimenticias, desarrollar nuevas alternativas y principalmente mejorar la calidad nutricional de los animales de consumo humano y generar a su vez patrones de producción ajustados a la realidad social y económica de cualquier entorno $(15,16)$, donde la producción de conejo resulta 
acorde por sus características fisiológicas y hábitos alimenticios que permiten adicionar en su dieta una gran variedad de recursos vegetales ya utilizados en otras especies de animales (17).

A pesar de todas las ventajas mencionadas y siendo el conejo productor potencial de carne a bajo costo con características de alto valor zootécnico como alta conversión alimenticia, animal porlífero de rápido crecimiento, facilidad de manejo y área de producción reducida; además de la textura, suavidad y sabor de su carne con niveles de proteínas adecuados a precios accesibles para el consumidor de escasos recursos económicos y aunado las potencialidades agronómicas ofrecidas por las regiones tropicales y subtropicales del mundo tales como Venezuela, la producción cunícola ha sido muy poco explotada.

De allí entonces, se hace necesaria la búsqueda de insumos que permitan establecer estrategias de alimentación no convencionales para la cunicultura, tales como follaje de mataratón (Gliricidia sepium) y fibra (cachaza o palmiste) de palma aceitera (Elaeis guineensis). El mataratón es una leguminosa arbustiva tropical que ofrece follaje fresco o deshidratado, con aportes importantes en la alimentación animal que incluyen proteínas (20.64-28.31\%), extracto etéreo $(2.93-4.80 \%)$ y cenizas $(8.88-7.40 \%)$ con minerales tales como $\mathrm{Ca}, \mathrm{Mg}, \mathrm{Zn}, \mathrm{Mn}, \mathrm{P}, \mathrm{K}$ y $\mathrm{Fe}(18)$. La cachaza de palma es el residuo sólido del prensado luego de la extracción del aceite de los frutos, cuyo aporte aproximado es de $5.3 \%$ en proteínas, $23.1 \%$ en grasa, $15.1 \%$ en fibra y $1.9 \%$ en cenizas (19). Ambos recursos han sido utilizados en la alimentación animal de monogástricos y son promisorios como suplemento en la elaboración de bloques multinutricionales (BMN) para la producción cunícola.

Con base en lo expuesto, el propósito del presente trabajo engloba estudiar comparativamente el valor nutricional de la carne de conejos alimentados con dietas suplementadas con follaje de mataratón y cachaza de palma aceitera, como recursos vegetales no comestibles por el hombre, con el fin de obtener datos relacionados con la composición bromatológica de este tipo de carne, prácticamente inexistentes en Venezuela, los cuales servirán para divulgar sus características nutricionales como alimento en pro de fomentar sus beneficios hacia una cultura de consumo que incentive una mayor producción de esta especie aprovechando estos recursos agronómicos locales.

\section{MATERIALES Y MÉTODOS}

Sitio de estudio y animales. Las muestras de carne analizadas en el presente estudio provienen de una investigación previa desarrollada en la Unidad cunícola del Fundo San Gregorio, Sector San Agustín vía El Barril, Parroquia La Pica, Municipio Maturín, Estado Monagas, Venezuela; ubicada a una altitud de $32 \mathrm{msnm}$ con las coordenadas entre $9^{\circ} 44^{\prime} 30.77^{\prime \prime}$ latitud Norte y $63^{\circ} 3^{\prime} 2.55^{\prime \prime}$ longitud Oeste; con clima ligeramente húmedo y cálido, con temperatura promedio de $28^{\circ} \mathrm{C}$, que varía de 23 a $35^{\circ} \mathrm{C}$, precipitación anual de $1340 \mathrm{~mm}$ con una distribución unimodal, evaporación de $1650 \mathrm{~mm}$ y humedad relativa media entre 67 y $85 \%$ durante todo el año (20).

Diseño experimental. Se utilizó un diseño experimental de bloques al azar con tres tratamientos (dietas) y tres repeticiones (bloques) con muestreo de tres réplicas por repetición con un lote de 27 conejos machos mestizos (California y Nueva Zelanda) nacidos en el mes de diciembre del año 2010 con poca diferencia de días.

Luego del destete, con edades entre 30 y 34 $(D E=1.68)$ días, los animales se asignaron al azar en grupos de tres por jaula, elevadas a una altura aproximada de $50 \mathrm{~cm}$ desde el suelo, conformando un total de nueve unidades experimentales, donde se les suministró el $A B C$ y los bloques multinutricionales (BMN) de $300 \mathrm{~g}$ suplementados con mataratón (Gliricidia sepium) y cachaza de palma aceitera (Elaeis guineensis), formulados de acuerdo con la tabla 1.

Tabla 1. Formulación de los BMN utilizados.

\begin{tabular}{lcc}
\hline \multicolumn{1}{c}{ Ingrediente } & $\mathbf{B M N}_{\mathbf{1}}$ & $\mathbf{B M N}_{\mathbf{2}}$ \\
\hline Melaza & $40 \%$ & $40 \%$ \\
Minerales & $10 \%$ & $10 \%$ \\
Cal viva & $5 \%$ & $5 \%$ \\
Heno & $5 \%$ & $5 \%$ \\
Mataratón & $30 \%$ & $10 \%$ \\
Cachaza & $10 \%$ & $30 \%$ \\
\hline
\end{tabular}

Durante el período comprendido entre los meses de enero y febrero del 2011, los animales se sometieron a las siguientes dietas (Tabla 2):

Tratamiento control $\left(\mathbf{T}_{0}\right)$. En base al alimento balanceado comercial (ABC) sin suplementación.

Tratamiento $\mathbf{1}\left(\mathbf{T}_{\mathbf{1}}\right)$. En base al $A B C$ suplementado $30 \%$ con mataratón y $10 \%$ con cachaza de palma aceitera.

Tratamiento $\mathbf{2}\left(\mathbf{T}_{\mathbf{2}}\right)$. En base al $A B C$ suplementado $10 \%$ con mataratón y $30 \%$ con cachaza. 
Tabla 2. Composición de las dietas utilizadas en la alimentación de los conejos.

\begin{tabular}{lccc}
\hline \multicolumn{1}{c}{ PARÁMETRO } & $\mathbf{T}_{\mathbf{0}} \mathbf{( \% )}$ & $\mathbf{T}_{\mathbf{1}}(\mathbf{\%})$ & $\mathbf{T}_{\mathbf{2}}(\mathbf{\%})$ \\
\hline Humedad & 10.60 & 10.34 & 10.41 \\
Proteína & 14.75 & 10.76 & 07.75 \\
Fibra & 16.62 & 10.17 & 13.70 \\
Grasa & 05.64 & 01.40 & 01.85 \\
Cenizas & 14.04 & 24.18 & 24.48 \\
Carbohidratos & 48.95 & 53.49 & 52.22 \\
\hline
\end{tabular}

Toma y conservación de muestras. Los tratamientos dietéticos de los animales concluyeron a las 11 semanas de edad con el subsecuente sacrificio de los mismos. Posterior al sacrificio, con un cuchillo de acero inoxidable las canales de cada animal se dividieron en mitades iguales, refrigeradas y luego transportadas al laboratorio para los análisis correspondientes.

Análisis de laboratorio. Todos los análisis se realizaron en los Laboratorios de Investigación del "Campus Juanico" perteneciente al Núcleo de Monagas de la Universidad de Oriente con sede en Maturín. Para este propósito, a las distintas media canal de cada animal se le extrajo el correspondiente soporte óseo, la grasa perirrenal y la subcutánea antes de la homogeneización de las diferentes muestras de carne con una picadora moulinex previo a la evaluación bromatológica, por triplicado a cada una de las réplicas, siguiendo las metodologías propuestas por la AOAC (21). Para la determinación de humedad se utilizó una estufa convencional a una temperatura de $102^{\circ} \mathrm{C}$ por 24 horas. Las cenizas se determinaron con una mufla a una temperatura de $550^{\circ} \mathrm{C}$ por un período de 4 horas. La determinación de proteínas se realizó por micro Kjeldahl y para los lípidos se empleó un soxhlet con una mezcla cloroformo/metanol 2:1 (v/v) como extractor para análisis gravimétrico (22).

Análisis de los resultados. Los datos obtenidos se estudiaron estadísticamente mediante un análisis de varianza, para determinar la existencia de diferencias significativas, con el programa Statistix versión 8.0 (23).

\section{RESULTADOS}

Debido al deceso de uno de los animales del total de 27, previo a la conclusión de los tratamientos dietéticos, los resultados están basados para los 26 conejos restantes, a manera de dilucidar la influencia de la suplementación en el estatus nutricional de la carne de los mismos. Luego de una evaluación de las diferentes muestras de carne, basada en la determinación de sus características bromatológicas (humedad, cenizas, proteínas y lípidos) y de acuerdo al análisis de varianza para el promedio de tres réplicas por repetición, se encontró que las carnes provenientes de los conejos en estudio no presentaron diferencias significativas $(p>0.05)$; cuyos resultados, mostrados en la tabla 3, corresponden al promedio general de todos los tratamientos con su respectiva desviación estándar (DE).

Tabla 3. Bromatología de las canales de los conejos.

\begin{tabular}{|c|c|c|c|}
\hline \multicolumn{4}{|c|}{ Parámetro Proximal $\%$ masa (promedio $\pm D E$ ) } \\
\hline Humedad & Cenizas & Proteínas & Lípidos \\
\hline $71.77 \pm 2.03$ & $1.62 \pm 0.19$ & $19.67 \pm 1.14$ & $6.94 \pm 1.74$ \\
\hline
\end{tabular}

De acuerdo con los resultados mostrados en la tabla 3, las carnes tienen una composición bromatológica con niveles de humedad entre 70.77 y $72.42 \%$, proteínas entre 19.08 y $20.34 \%$, cenizas entre 1.53 y $1.68 \%$ y lípidos entre 6.48 y $7.23 \%$.

\section{DISCUSIÓN}

Dado que en Venezuela no existe información relacionada con los parámetros evaluados en el presente trabajo, la discusión se basará en reportes para experimentos llevados a cabo en otras latitudes del mundo. Como se mencionó anteriormente en los resultados, con base en el análisis de varianza, se encontró que la suplementación no afectó significativamente la composición bromatológica de las carnes provenientes de los conejos en estudio; por lo tanto, se puede inferir que estas carnes son nutricionalmente idénticas como alimento para el consumo humano, indistintamente de la dieta a base del $A B C$ solo o suplementado con mataratón/cachaza de palma aceitera, lo cual los hace adecuados como recursos agronómicos de suplementación dietética para la cunicultura, con un posible ahorro en los costos de producción.

Humedad. Con base en diferentes estudios realizados, algunos autores refieren que la composición de la carne está estrechamente relacionada con la edad del animal observándose que la humedad disminuye con el aumento de la edad (7); por tanto, la edad de los animales no fue un factor de variabilidad para este parámetro, ya que los animales estudiados poseían edades similares. Los valores de humedad obtenidos son similares a los reportados por Gašperlin et al (24), para animales entre 77 y 90 días, con porcentajes alrededor de $72.7 \%$ para el mismo músculo; y son un poco más bajos que la mayoría de los reportes con valores aproximados de $74.30 \%(25), 74.36 \%$ (26) $75.07 \%$ (7), 
$75.56 \%$ (27). Entre los estudios más recientes se puede destacar el de Meineri et al (25), quienes utilizaron las semillas de chía (Salvia hispanica L.) como suplemento de las dietas, en $10 \%$ y $15 \%$, obteniéndose carnes con calidad similar al control. Simonová et al (7) obtuvieron resultados parecidos al utilizar una suplementación con fitoaditivos tales como orégano.

Cenizas. De acuerdo a los resultados de la tabla 3, los valores de cenizas obtenidos en este estudio superan la gran mayoría de los reportes más recientes de algunos autores, quienes encontraron contenidos entre $1.07 \mathrm{y}$ $1.43 \%(7,25,26)$. Las cenizas representan el contenido relativo de minerales que pueden incluir cualquiera de los iones esenciales para los humanos; haciendo posible decir, que las carnes de la presente investigación tienen un aporte nutricional suficiente con respecto al suministro de minerales.

Proteínas. Los resultados obtenidos para el contenido de proteínas en este estudio, son similares a los reportados por Vieira De Sousa (27) y Dalle Zotte (12) de 19.37 y $20.05 \%$ respectivamente; siendo a su vez un poco menor a los presentados por Cury et al (28), 20.95, Pascual et al (26), 20.96\%, Ariño (29), $21.00 \%$, Simonová et al (7), 21.53\%, Gašperlin et al (24), $22.15 \%$ y Meineri et al (25), $22.33 \%$; lo cual podría ser debido a las diferencias en la edad o pesos de los animales estudiados por estos autores. Si se estudian los pesos individualmente, se puede observar que los animales con peso desde 2.10 a $2.20 \mathrm{Kg}$ reportan una menor cantidad de proteína con respecto a los que poseen pesos superiores a los $2.25 \mathrm{Kg}$, como se muestra en la tabla 4 (20).

Tabla 4. Peso de los conejos y contenido de proteínas de las canales por tratamiento.

\begin{tabular}{cccccc}
\hline \multicolumn{2}{c}{ Control $\left(\mathbf{T}_{\mathbf{0}}\right)$} & \multicolumn{2}{c}{ Tratamiento $\mathbf{1}\left(\mathbf{T}_{\mathbf{1}} \mathbf{)}\right.$} & \multicolumn{2}{c}{ Tratamiento $\mathbf{2}\left(\mathbf{T}_{\mathbf{~}} \mathbf{)}\right.$} \\
\hline $\mathbf{K g}$ & \% Proteínas & $\mathbf{K g}$ & \% Proteínas & $\mathbf{K g}$ & \% Proteínas \\
2.46 & 21.04 & 2.40 & 17.55 & 2.44 & 21.25 \\
2.39 & 21.19 & 2.40 & 17.04 & 2.44 & 20.96 \\
2.37 & 20.71 & 2.17 & 19.10 & 2.44 & 20.09 \\
2.28 & 19.19 & 2.17 & 20.50 & 2.44 & 19.82 \\
2.27 & 20.21 & 2.12 & 18.57 & 2.44 & 19.24 \\
2.18 & 19.87 & 1.96 & 21.13 & 2.44 & 19.23 \\
2.17 & Deceso & 1.95 & 19.85 & 2.44 & 18.65 \\
2.16 & 20.98 & 1.95 & 18.58 & 2.44 & 18.62 \\
2.05 & 19.50 & 1.81 & 19.39 & 2.44 & 18.29 \\
\hline
\end{tabular}

Lípidos. En cuanto al contenido de lípidos, de acuerdo a la tabla 3, los valores de esta investigación son mayores a los porcentajes ya reportados en trabajos previos para las canales de estos animales, entre 0.97 y $4.10 \%$ $(7,12,25,26,30)$; lo cual podría ser debido a que en dichos trabajos al emplear únicamente solventes no polares, se logra extraer sólo a los lípidos no polares constituidos mayormente por triglicéridos que son reportados como grasas; a diferencia de la presente investigación, donde se realizó la extracción usando una mezcla de solventes cloroformo/metanol 2:1 (v/v), haciendo posible extraer a los lípidos compuestos con fragmentos polares, tales como fosfolípidos, además de los lípidos no polares o neutros, que incluyen triglicéridos y ésteres de colesterol (22).

En las investigaciones más recientes, a través de la manipulación de las dietas de los conejos, se están utilizando diferentes estrategias con la finalidad de producir carne funcional y productos cárnicos funcionales logrando efectividad en el incremento de los niveles de ácidos grasos poliinsaturados $\omega-3(5,31)$ e incluso de la estabilidad oxidativa de la carne usando vitamina E o aceites vegetales $(32,33)$, con lo que se vislumbra, sin lugar a dudas, que la carne de conejo se fortalecerá aún más dentro de los hábitos alimenticios saludables para los humanos.

Con base a los resultados obtenidos en el presente estudio, se puede indicar que las carnes provenientes de los conejos, sometidos tanto a la dieta control (sólo con $A B C$ ) como a las dietas suplementadas ( $A B C$ con follaje de mataratón/ cachaza de palma aceitera), son nutricionalmente idénticas como alimento para el consumo humano; sugiriéndose así, que es posible continuar utilizando estos recursos agronómicos como suplemento dietético en la experimentación cunícola orientada hacia la mayor disminución en los costos de producción, a manera de que aquellos con escasos recursos económicos también tengan acceso a los amplios beneficios dietéticos, que como alimento catalogan a esta carne, tanto a nivel nutricional como saludable $(31,33,34)$.

En conclusión, se recomienda continuar con las investigaciones relacionadas con el uso de estos mismos recursos agronómicos, mataratón y fibra de palma aceitera explorando otras dietas incluso sin alimento comercial, con el fin de encontrar la opción más viable y rentable para la explotación cunícola a manera de continuar con los estudios nutricionales de estas carnes.

\section{Agradecimiento}

Al Consejo de Investigación de la Universidad de Oriente, Venezuela, por el apoyo brindado a la presente investigación a través del proyecto CI-4010201-1325/06. 


\section{REFERENCIAS}

1. Delisle HF, Vioque J, Gil A. Dietary patterns and quality in West-African immigrants in Madrid. Nutr J 2009; 8(3):1-10. URL Disponible en: http://www.nutritionj.com/ content/pdf/1475-2891-8-3.pdf.

2. Mente A, Koning L, Shannon $H$, Anand S. A systematic review of the evidence supporting a causal link between dietary factors and coronary heart disease. Arch Intern Med 2009; 169(7):659-669.

3. Yahia N, Achkar A, Abdallah A, Rizk S. Eating habits and obesity among Lebanese university students. Nutr J 2008; 7(32):1-6. URL Disponible en: http://www.nutritionj. com/content/pdf/1475-2891-7-32.pdf .

4. Schönfeldt H, Gibson N. Changes in the nutrient quality of meat in an obesity context. Meat Sci 2008; 80(1):20-27.

5. Hernández P. Enhancement of nutritional quality and safety in rabbit meat. In: Xiccato G, Trocino A, Lukefahr S editors. 9th World Rabbit Congress. World Rabbit Sciences: meat quality and safety; Verona june 10-13, 2008. Brescia - Italy: Fondazione Iniziative Zooprofilattiche e Zootecniche; 2008.

6. Hernández $P$, Dalle Zotte $A$. Influence of diet on rabbit meat quality. In: De Blas, C.; Wiseman, J. Nutrition of the rabbit. 2nd ed. Oxfordshire: CAB International; 2010.

7. Simonová M, Chrastinová L, Mojto J, Lauková A, Szabóová R, Rafay J. Quality of rabbit meat and phyto-additives. Czech J Food Sci 2010; 28(3):161-167.

8. Ariño $B$, Hernández $P$, Pla $M$, Blasco $A$. Comparison between rabbit lines for sensory meat quality. Meat Sci 2007; 75(3):494-98.

9. Cavani C, Petracci M, Trocino A, Xiccato G. Advances in research on poultry and rabbit meat quality. Ital J Anim Sci 2009; 8 Suppl2:741-750.

10. Polak $T$, Gašperlin L, Rajar A, Žlender B. Influence of genotype lines, age atslaughter and sexes on the composition of rabbit meat. Food Technol Biotechnol 2006; 44(1):65-73.
11. Hermida M, González M, Miranda $M$, Rodríguez-Otero JL. Mineral analysis in rabbit meat from Galicia (NW Spain). Meat Sci 2006; 73(4):635-639.

12. Dalle Zotte A. Perception of rabbit meat quality and major factors influencing the rabbit carcass and meat quality. Livest Prod Sci 2002; 75(1):11-32.

13. Hernández, P. 2007. Carne de conejo, ideal para dietas bajas en ácido úrico. Revista Científica de Nutrición. Bol Cunicul 154(8):33-36.

14. Combes S. Nutritional value of rabbit meat: a review. INRA Prod Anim 2004; $17(5): 373-383$.

15. Nieves D. Forrajes promisorios para la alimentación de conejos en Venezuela. Valor nutricional. Guanare, Venezuela: Universidad Ezequiel Zamora; 2005.

16. Nieves $D$, Terán $O$, Vivas $M$, Arciniegas G, González C. Comportamiento productivo de conejos alimentados con dietas basadas en follajes tropicales. Rev Científ FCV-LUZ 2009; 21(2):173-180.

17. Dihigo L. Avance en los estudios de fisiología digestiva del conejo en Cuba con el uso de fuentes de alimentos no tradicionales. Consideraciones fisiológicas. [En linea]. Instituto de Ciencia Animal, La Habana, Cuba, 2006. (consultado 05/10/2011). URL Disponible en: http://www.sian.info. ve/porcinos/publicaciones/encuentros/ viii_encuentro/luise.htm

18. Araque $C$, Quijada $T, D^{\prime}$ Aubeterre $R$, Páez L, Sánchez A, Espinoza F. Bromatología del mataratón (Gliricidia sepium) a diferentes edades de corte en Urachiche, Estado Yaracuy, Venezuela. Zootecnia Trop 2006; 24(4):393-399.

19. Ocampo A. La palma aceitera africana, un recurso de alto potencial para la producción animal en el trópico. [En linea] 2012. (Consultado: 20/04/2012). Disponible en: http://www.fao.org/docrep/V4440T/ v4440T0g.htm 
20. Urpín, L. Sustitución parcial del alimento balanceado por bloques multinutricionales elaborados con mataratón (Gliricidia sepium) y fibra del fruto de palma aceitera (Elaeis guineensis) en la alimentación de conejos mestizos. [Tesis]. Maturín, Venezuela: Universidad de Oriente, Programa de Ingeniería en Producción Animal, Núcleo de Monagas; 2012.

21. AOAC International. Official Methods of Analysis. Ass Agric Chem $18^{\text {th }}$ ed. Washigton: AOAC; 2005.

22. Mariezcurrena MA, Braña D, Partida J, Ramírez E, Domínguez I. Estandarización de la metodología para la determinación de grasa en la carne de cerdo. Rev Mex Cienc Pecu 2010; 10(3):269-275.

23. Statistix versión 8.0. Statistix.com. Documento en línea (Consultado: 12/03/2012). URL Disponible en: http:// www.statistix.com

24. Gašperlin L, Polak T, Rajar A, Skvarèa $M$, Lender B. Effect of genotype, age at slaughter and sex on chemical composition and sensory profile of rabbit meat. World Rabbit Sci 2006; 14(3):157-166.

25. Meineri G, Cornale P, Tassone S, Peiretti P. Effects of Chia (Salvia hispanica L.) seed supplementation on rabbit meat quality, oxidative stability and sensory traits. Ital J Anim Sci 2010; 9(10):45-49.

26. Pascual M, Aliaga S, Pla M. Effect of selection for growth rate on carcasses and meat composition in rabbits. Proceedings of the $8^{\text {th }}$ World Rabbit Congress. World Rabbit Sciences: meat quality and safety. Puebla, Mexico. Spain: University of Valencia; 2004.
27. Vieira De Sousa D. Características de qualidade da carne de coelhos alimentados com rações contendo farelo de coco. [Tesis de Mestrado]. Forza, Brazil: Universidade Federal do Ceará; 2007. URL Disponible en: http://www.ppgcta.ufc.br/danielasouza.pdf

28. Cury K, Martínez A, Aguas Y, Oliveros R. Caracterización de carne de conejo y producción de salchicha. Rev Colombiana Cienc Anim 2011; 3(2):269-282.

29. Ariño B. Variabilidad genética de la calidad de la carne de conejo. [Tesis Doctoral]. Valencia, España: Universidad Politécnica de Valencia; 2006. URL Disponible en: http://riunet. upv.es/bitstream/handle/10251/5642/ tesisUPV2479.pdf? sequence $=1$

30. Ramírez J, Díaz I, Pla M, Gil M, Blasco A, Oliver M. Effect of selection for growth rate on biochemical, quality and texture characteristics of meat from rabbits. Food Chem 2005; 90(1-2):251-256.

31. Hernández, P. Carne de conejo como alimento funcional. Cunicultura 2012; 37(218):21-24.

32. Nuchi C, Magrinyá N, Tres A, Bou R, Guardiola F, Codony R. Results on lipid composition and oxidation in animal samples. [En linea] 2007. (Consultado: 20/07/2012). URL Disponible en: http:// www.ub.es/feedfat

33. Dalle Zotte $A$, Zcendro $Z$. The role of rabbit meat as functional food. Meat Sci 2011; 88(3):319-331.

34. Malavé A, Coronado L, Noriega A, Figuera Y. Propiedades dietéticas de la carne de conejo en la nutrición y salud humana. Rev Cient UDO Agr in press 2013. 